\title{
An Overview of Definition and Approaches to Petroclival Meningioma
}

\author{
Alireza Khoshnevisan* \\ Department of neurosurgery, Tehran university of medical sciences, Tehran, Iran \\ *Corresponding author: Alireza Khoshnevisan, Associate professor of neurosurgery, Tehran university of medical sciences, Tehran, \\ Iran
}

\begin{abstract}
Meningiomas account for 20 to $25 \%$ of all intracranial tumors and $10 \%$ are seen in the posterior fossa. Petroclival tumors are defined as arising from the upper two thirds of the clivus, medial to the trigeminal nerve at the petroclival junction. Brain CT scan and MRI are routinely used to evaluate tumor anatomy. The petrous temporal bone is a hindrance to resection of these tumors. Oftentimes neurosurgeons are unskilled with the anatomy of the petrous bone, and so suboccipital and pterional routes are usually preferred. Some authors have encouraged using traditional neurosurgical approaches to these tumors. In this manuscript we review tumor location and approaches used for resection of these challenging tumors.
\end{abstract}

Keywords: Meningioma; Petroclival; Approaches

Abbreviations: IAM: Internal Auditory Meatus; GTR: Gross total resection; NTR: Near total resection; STR: Subtotal resection.

\section{Introduction}

Meningiomas account for 20 to $25 \%$ of all intracranial tumors and $10 \%$ are seen in the posterior fossa. Of posterior fossa meningiomas, those rising from the petroclival region account for 5 to $11 \%$ of meningiomas and thus $0.15 \%$ of all intracranial tumors. Male to female ration is 1 to 4 . Petroclival tumors are defined as arising from the upper two thirds of the clivus, medial to the trigeminal nerve at the petroclival junction, often extent to the middle and approaches posterior cranial fossae, and can involve the posterior cavernous sinus through the Meckel cave [1].

Growth rates were $0.81 \mathrm{~mm} / \mathrm{yr}$ (Diameter) and $0.81 \mathrm{~cm} 3 /$ yr (Volume). When only growing tumors were measured, the mean growth rates were $1.16 \mathrm{~mm} / \mathrm{yr}$ (Diameter) and $1.10 \mathrm{~cm} 3$ / yr (Volume). Untreated tumors may grow at a dissimilar rate than resected tumors [2]. The petrous temporal bone is a hindrance for tumor resection in this location. Oftentimes neurosurgeons are unskilled with the anatomy of the petrous bone, and so suboccipital and pterional routes are preferred. Some authors have encouraged using traditional neurosurgical approaches to these tumors.

\section{Discussion}

To select an appropriate approach all patients typically undergo CT to examine the bony architecture and relationships between the skull base and tumor. MRI is routinely used to evaluate presence of edema in the brainstem and soft-tissue relationships between the CNS and the tumor. Angiography to evaluate the arterial supply and relationship to major vessels is sometimes useful. Selection of approach depends on location, size, extension of the tumor, venous sinus anatomy and preoperative hearing evaluation.

Strategies to overcome the petrous temporal bone as an obstacle are:

a) Resection of the petrous apex with the middle fossa approach.

b) Resection of presigmoid, retrolabyrinthine petrous bone with the posterior petrosal approach.

c) Resection of the entire petrous bone with complete petrosectomy. 
Mayberg and Symon believed that the combined sub temporalposterior fossa approach provided the best results, as did Guthrie et al. Various alterations of this approach have been described by Samii et al. and Hakuba et al. Al-Mefty and co-workers have described in detail a petrosal approach for these tumors [3].

Tumors that are medial to the internal auditory canal and extent to both middle and posterior cranial fossae are often best treated with a combined transpetrosal approach, which is particularly true if there is a preoperative hearing deficit. The anterior petrosal approach is best appropriate for smaller petroclival meningiomas that do not spread lateral to the IAM. Once a tumor has extended farther into the posterior fossa, lateral to the IAM, a posterior petrosal approach should be used. A retrosigmoid craniotomy remains a workhorse surgical approach for most petroclival tumors of any size (Figure 1).

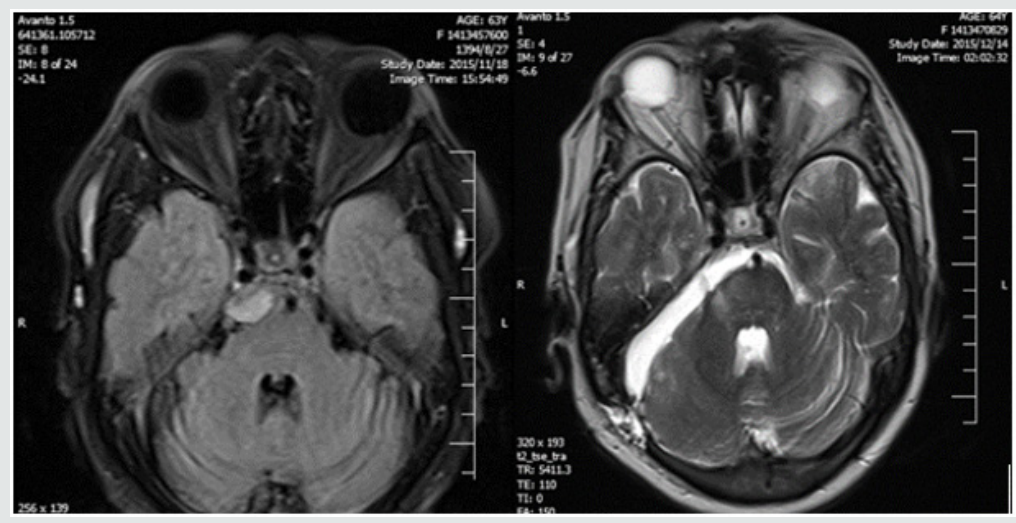

Figure 1: Pre and post-operative MRI of small right petrocloval meningioma resected by retrosigmoid approach.

More Recently, the Endoscopic Endonasal Approach has been "expanded" to the petroclival region. This approach should be favored for extradural midline tumors (chordomas, chondrosarcomas) or for cystic lesions when drainage is crucial [4]. GTR is only reported in an average of $49 \%$ of patients. Many highvolume surgical centers have abandoned GTR for NTR or STR later in their practice. Most describe that STR/NTR does not have an increased risk or recurrence compared with GTR in most series [5].

Attempts at accomplishing gross- or near-total resections are associated with an increased rate of morbidity and mortality. (Adjunctive treatment options such as stereotactic radiosurgery is attractive options). There has been a tendency to accept subtotal resections in an effort to minimize neurological morbidity [6]. Radiation therapy is used if a satisfactory decompression cannot be implemented or there is regrowth after radical subtotal removal [3].

Motor deficits and facial nerve morbidity are the common postoperative neurological complications. CSF leak occur in $10 \%$ of patients. In spite of the high morbidity, $>75 \%$ of patients return to independence at 1 year, many of which will start again employment [5].

\section{Conclusion}

Petroclival tumors also infrequent still remain a surgical challenge. The petrous temporal bone is an obstacle for tumor resection in this location. Oftentimes neurosurgeons are unfamiliar with the anatomy of the petrous bone, and so suboccipital and pterional routes are used. Some authors have advocated using traditional neurosurgical approaches to these tumors. The infrequency of the tumor, different treatment philosophies, and variations in reporting, complicate the outcome analysis and so choosing a definite approach for these tumors.

\section{References}

1. H Richard Winn (2017) Youmans \& Winn neurological surgery 4-Volume Set, 7th Edition. Elsevier Philadelphia, USA, Pp: 1108.

2. Daniel M Fountain, Wai Cheong Soon, Tomasz Matys, Mathew R Guilfoyle, Ramez Kirollos, et al. (2017) Volumetric growth rates of meningioma and its correlation with histological diagnosis and clinical outcome: a systematic review. Acta Neurochir (Wien) 159 (3): 435-445.

3. Ojemann Robert G (1992) Convexity Meningiomas. Clinical Neurosurgery. 40(17): 321-383.

4. Timothée Jacquesson, Moncef Berhouma, Stéphane Tringali, Emile Simon, Emmanuel Jouanneau, (2015) Which routes for petroclival tumors? A comparison between the anterior expanded endoscopic endonasal approach and lateral or posterior routes. World Neurosurgery 83(6): 929-936.

5. Michael L DiLuna, Ketan R Bulsara (2010) Surgery for Petroclival Meningiomas: A Comprehensive Review of Outcomes in the Skull Base Surgery Era. Skull Base 20(5): 337-342.

6. Feng $\mathrm{Xu}$, Ioannis Karampelas, Cliff A Megerian, Warren R Selman, Nicholas C Bambakidis (2013) Petroclival meningiomas: an update on surgical approaches, decision making, and treatment results. Neurosurg Focus 35(6): E11. 
(C) (i) This work is licensed under Creative

To Submit Your Article Click Here: Submit Article

DOI: $10.32474 / 0 J N B D .2020 .04 .000194$

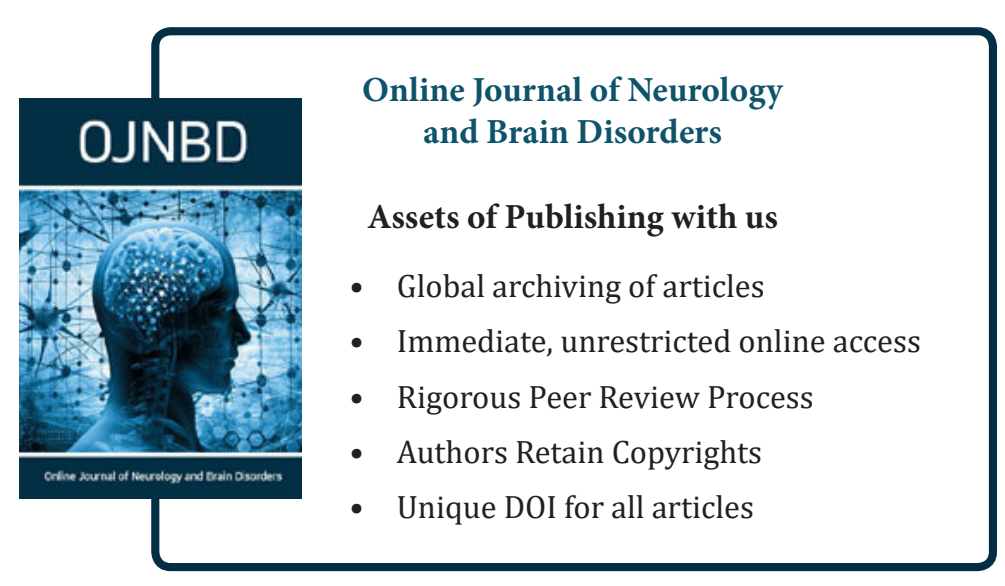

